\title{
Nicht rauchen und gewinnen
}

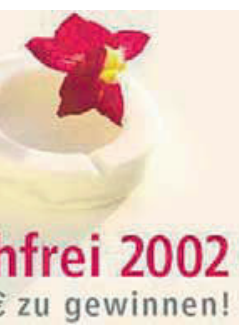

aucher, die
mindestens vom 1. bis 29. Mai auf ihr Laster verzichten, können gewinnen: Viermal 2.500 Euro verlost das Deutsche Krebsforschungszentrum im Rahmen der Kampagne „Rauchfrei 2002“ unter den Ausstei- gern. Teilnehmer können sich über das Internet (www.dkfz.de/rauchfrei2002) oder über Teilnahmekarten, die in Praxen, Apotheken und Kliniken ausliegen, anmelden. Ärzte, die die Aktion unterstützen wollen, können entsprechende Materialien bestellen beim DKFZ, Stabstelle Krebsprävention, Im Neuenheimer Feld 280, D-69120 Heidelberg, Fax: 06221 / 423020 oder per E-mail: Rauchfrei2002@dkfz.de.

\section{Welt-Asthmatag am 7. Mai 2002}

D er Welt-Asthmatag, der im Jahr 1998 zum ersten Mal begangen wurde, findet dieses Jahr am 7. Mai statt. Ins Leben gerufen haben diese Kampagne die WHO und die „Global Initiative for the Treatment of Asthma“" (GINA). Ihr Anliegen ist es, durch mehr Information über Therapiemöglichkeiten bei Asthma den Betroffenen zu helfen, besser mit der Erkrankung zurecht zu kommen. In Deutschland beteiligen sich an der Aktion der Deutsche Allergie- und Asthmabund e. V., die Germany Airway League und die Working Group of Paediatric Pneumology and Allergology. Waren es 1998 erst 35 Länder, die sich am Welt-Asthmatag beteiligten, sind es jetzt bereits über 90 .

mwe

\section{Die „Allergica“ lädt ein}

$\mathrm{V}$ om 24. bis 26. Mai findet erstmals in Frankfurt die neue Messe „Allergica" statt. Sie umfasst einen medizinischen Fachkongress, eine Fachausstellung und ein Informationsforum und richtet sich damit sowohl an den Fachbesucher aus dem medizinischen Bereich als auch an die Betroffenen selbst. Schirmherrin ist Bundesgesundheitsministerin Ulla Schmidt.

Aufgabe der Messe ist u.a. die zielgruppenorientierte Ansprache von Arzt, Patient und Öffentlichkeit. Man will ein Forum für interdisziplinäre Strategien schaffen und sinnvolle Kooperationen mit dem Ziel der Prävention und Kostendämpfung initiieren.

Weitere Informationen zu Anmeldung, Anreise und Programmablauf sind im Internet unter der Adresse www.allergica.de abrufbar.

\section{Pollenflug immer länger und intensiver}

$S_{\mathrm{eit}}^{\mathrm{c}}$ eit Jahren werden in den meisten europäischen Ländern penibel Pollenflugkalender geführt und bei der EAN, dem europäischen PollenflugNetzwerk, gesammelt. Mehr als 20.000 Jahresberichte der EAN aus den Jahren 1974 bis 2001 zeigten, dass sich Start und Höhepunkt der Pollensaison bei

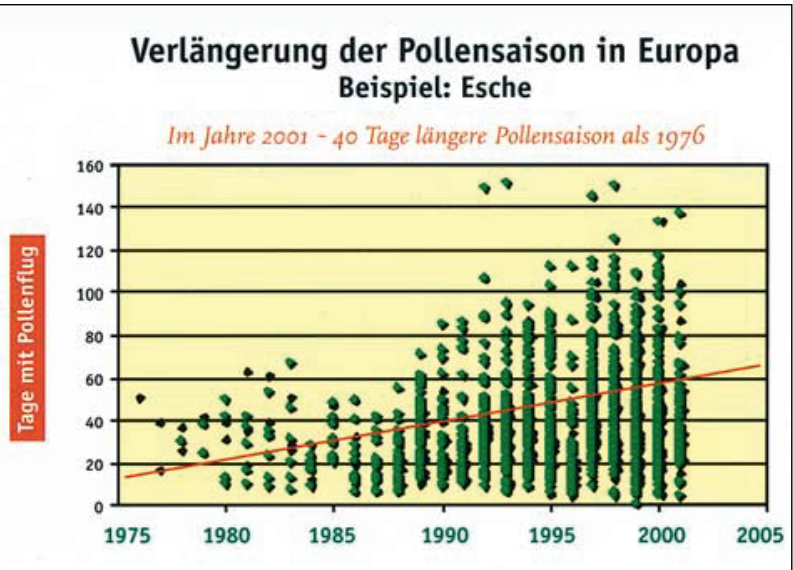

den Frühblühern wie Erle, Hasel, Weide und Ulme sich im Schnitt um 20 Tage nach vorne geschoben haben.

Zudem ist eine verlängerte Pollenflugsaison zu beobachten, in Deutschland insbesondere bei Gräsern und Kräutern, aber auch bei Bäumen, die zwischen März und Mai blühen, wie z.B. die Esche. Bei Erle und Hasel, aber auch Weide und Ulme konnte dieser Trend in den letzten Jahrzehnten nicht gezeigt werden. Eine insgesamt höhere Pollenbelastung zeigte sich bei Pflanzen-Gattungen, die zwischen Juni und September blühen. Dazu zählen u.a. Gräser und Getreide, aber auch Gänsefuß, Sauerampfer und Spitzwegerich. $\quad f k$

Presseinformation des ÄDA und der DGAI

\section{Polleninformations- dienst sucht Mikroskop!}

$\mathrm{n}$ einer langjährig praktizierenden Pollenmessstation der Stiftung Deutscher Polleninformationsdienst wurde das Mikroskop gestohlen! Die Pollenzählungen dort sind deshalb nicht mehr gewährleistet. Der Vorstandsvorsitzender Prof. Dr. K.-Ch. Bergmann hat darum die kollegiale Bitte zu prüfen, ob ein Allergo-Journal-Leser oder eine Klinik der Stiftung ein nicht mehr benötigtes Mikroskop zur Verfügung stellen kann. Das Mikroskop muss eine 10er und eine 40er Optik (=500fache Vergrößerung) enthalten. Die Stiftung stellt gerne eine Spendenbescheinigung aus.

Kontakt: Stiftung Deutscher Polleninformationsdienst, Frau Margarete Wilhelm, Burgstr. 12,

D-33175 Bad Lippspringe

Tel.: 05252 / 931203

Fax: 05252 / 954501

E-mail: pollenstiftung@t-online.de 